\title{
SLIP EFFECTS ON SQUEEZING FLOW OF NANOFLUID BETWEEN TWO PARALLEL DISKS
}

\author{
K. DAS \\ Department of Mathematics, A.B.N. Seal College \\ Cooch Behar, Pin-736101, INDIA \\ E-mail:kd_kgec@rediffmail.com \\ S. JANA \\ Department of Mathematics, Jadavpur University \\ Kolkata 70003, INDIA \\ E-mail: jana05subrata@gmail.com \\ N. ACHARYA \\ Department of Mathematics, P.R. Thakur Govt. College \\ Gaighata, 24 Pgs(N), W.B., INDIA \\ E-mail: nilangshu.math@gmail.com
}

\begin{abstract}
In this study, the influence of temperature and wall slip conditions on the unsteady flow of a viscous, incompressible and electrically conducting nanofluid squeezed between two parallel disks in the presence of an applied magnetic field is investigated numerically. Using the similarity transformation, the governing coupled partial differential equations are transformed into similarity non-linear ordinary differential equations which are solved numerically using the Nachtsheim and Swigert shooting iteration technique together with the sixth order Runge -Kutta integration scheme. The effects of various emerging parameters on the flow characteristics are determined and discussed in detail. To check the reliability of the method, the numerical results for the skin friction coefficient and Nusselt number in the absence of slip conditions are compared with the results reported by the predecessors and an excellent agreement is observed between the two sets of results.
\end{abstract}

Key words: nanofluid, slip effect, squeezing flow, magnetic field.

2010 Mathematics Subject Classification. 76W05

\section{Introduction}

In recent years, the study of heat and mass transfer of viscous fluids in the squeezing flow has been increased due to their applications in many branches of science and engineering. Such flows are induced by two approaching parallel surfaces in relative motion. The use of on MHD fluid in lubrication prevents the adverse impact of temperature on the fluid viscosity when the system operates under extreme conditions. Stefan [1] first initiated pioneering work on the squeezing flow by invoking a lubrication approach. Rashidi et al. [2] studied the two-dimensional and axisymmetric squeezing flow between parallel plates. The effect of magnetic field in the squeezing flow between infinite parallel plates due to the normal motion of plates was discussed by Siddiqui et al. [3]. Domairry and Aziz [4] provided the approximate analytic solution for the squeezing flow of a viscous fluid between parallel disks with suction or blowing. Hayat et al. [5] extended

\footnotetext{
* To whom correspondence should be addressed
} 
the work presented in Ref. [4] to analyze the squeezing flow of a second grade fluid between parallel disks. Recently, Mustafa et al. [6] discussed the squeezing flow of a viscous fluid between two parallel plates.

In the recent past a new class of fluids, namely nanofluids, have attracted the attention of the engineering community. An innovative way of improving the thermal conductivities of heat transfer fluids is to suspend small solid particles in the fluids. Nanofluids are nanometer-sized particles dispersed in a base fluid such as water, ethylene glycol, toluene and oil. The addition of high thermal conductivity metallic nanoparticles increases the thermal conductivity of such mixtures; thus enhancing their overall energy transport capability. Nanofluids have properties that make them potentially useful in many heat transfer processes including microelectronics, fuel cells, pharmaceutical processes and hybrid-powered engines. They exhibit enhanced thermal conductivities and heat transfer coefficients compared to the base fluids. For this reason nanofluids are often preferred to conventional coolants like oil, water, ethylene glycol mixtures. The enhancement of thermal conductivities by nanofluids was first discussed by Choi [7]. Subsequently, Choi et al. [8] showed that the addition of a small amount of nanoparticles to conventional fluids (less than $1 \%$ by volume) approximately doubled the thermal conductivity of the liquid. It should be noticed that there have been published several recent papers [9-16] on the mathematical and numerical modeling of convective heat transfer in nanofluids. Kandasamy et al. [17] investigated an MHD boundary layer flow of a nanofluid over a stretching surface using scaling group transformation. Hashmi et al. [18] provided analytical solutions for the squeezing flow of nanofluids between parallel disks in the presence of a magnetic field. Recently, Das [19] studied the stagnation point nanofluid flow in the presence of chemical reaction and magnetic field using the Lie group analysis.

In these studies the fluid velocity is assumed to be zero relative to the solid boundary. But this is not true for fluid flows at the micro and nano scale. Investigation shows that a slip flow happens when the characteristic size of the flow system is small or the flow pressure is very low. A partial slip may occur on a stationary and moving boundary when the fluid is particulate such as emulsions, suspensions, foams, and polymer solutions. To describe the phenomenon of slip, Navier [20] introduced a boundary condition which states that the component of the fluid velocity tangential to the boundary walls is proportional to tangential stress. Later, several researchers [21-23] extended the Navier boundary conditions. A number of significant studies [24-28] have been done analytically and numerically regarding the slip flow regimes over different types of flow configurations. These results demonstrated that the boundary layer equation can be used to study the flow at the micro electro mechanical system (MEMS) scale and provide useful information to study the effects of rarefaction on the shear stress and structure of the flow. The magnetohydrodynamic squeezing flow of nanofluids between parallel disks was performed by Hussain et al. [29]. Recently, Das [30] analyzed the convective heat transfer characteristic of nanofluid over a permeable stretching surface with partial slip velocity.

The author's best knowledge, a study on an unsteady MHD squeezing flow of a nanofluid between two parallel disks with velocity slip and temperature jump has never been made till date. Therefore, the objective of the present paper is to investigate the slip effects on the squeezing flow of a nanofluid between two parallel disks in the presence of a magnetic field.

\section{Mathematical formulation of the problem}

Let us consider an axisymmetric flow of an incompressible electrically conducting viscous nanofluid between two parallel disks separated by a variable distance $h(t)=H(1-\alpha t)^{I / 2}$ where $\alpha$ is a characteristic parameter having dimensions of time inverse. The upper disk at $z=h(t)$ is approaching the lower stationary disk at $z=0$ with a velocity $v(t)=d h / d t$ until they touch. A uniform magnetic field of strength $B(t)=B_{0}(1-\alpha t)^{-1 / 2}$ is applied perpendicular to the disks as shown in Fig.1. The magnetic Reynolds number is assumed to be small so that the induced magnetic field can be neglected. The fluid structure is everywhere in local thermodynamic equilibrium. The plate is maintained at a constant temperature. 


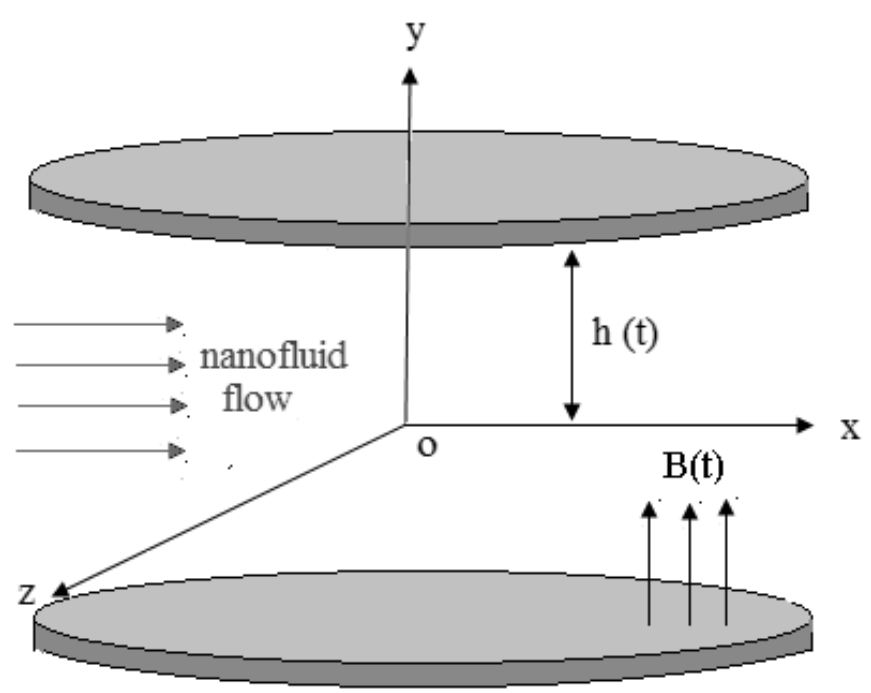

Fig.1. Physical model of the problem. expressed as

Under the stated assumptions, the governing conservation equations at unsteady state can be

$$
\begin{aligned}
& \frac{\partial u}{\partial r}+\frac{u}{r}+\frac{\partial w}{\partial z}=0, \\
& \frac{\partial u}{\partial t}+u \frac{\partial u}{\partial r}+w \frac{\partial u}{\partial z}=-\frac{1}{\rho_{f}} \frac{\partial p}{\partial r}+v\left(\frac{\partial^{2} u}{\partial r^{2}}+\frac{\partial^{2} u}{\partial z^{2}}+\frac{1}{r} \frac{\partial u}{\partial r}-\frac{u}{r^{2}}\right)-\frac{\sigma B^{2}(t)}{\rho_{f}} u, \\
& \frac{\partial w}{\partial t}+u \frac{\partial w}{\partial r}+w \frac{\partial w}{\partial z}=-\frac{1}{\rho_{f}} \frac{\partial p}{\partial z}+v\left(\frac{\partial^{2} w}{\partial r^{2}}+\frac{\partial^{2} w}{\partial z^{2}}+\frac{1}{r} \frac{\partial w}{\partial r}\right), \\
& \frac{\partial T}{\partial t}+u \frac{\partial T}{\partial r}+w \frac{\partial T}{\partial z}=\alpha\left(\frac{\partial^{2} T}{\partial r^{2}}+\frac{1}{r} \frac{\partial T}{\partial r}+\frac{\partial^{2} T}{\partial z^{2}}\right)+ \\
& +\tau\left[D_{B}\left(\frac{\partial C}{\partial r} \frac{\partial T}{\partial r}+\frac{\partial C}{\partial z} \frac{\partial T}{\partial z}\right)+\frac{D_{T}}{T_{m}}\left\{\left(\frac{\partial T}{\partial r}\right)^{2}+\left(\frac{\partial T}{\partial z}\right)^{2}\right\}\right], \\
& \frac{\partial C}{\partial t}+u \frac{\partial C}{\partial r}+w \frac{\partial C}{\partial z}=D_{B}\left(\frac{\partial^{2} C}{\partial r^{2}}+\frac{1}{r} \frac{\partial C}{\partial r}+\frac{\partial^{2} C}{\partial z^{2}}\right)+\frac{D_{T}}{T_{m}}\left(\frac{\partial^{2} T}{\partial r^{2}}+\frac{1}{r} \frac{\partial T}{\partial r}+\frac{\partial^{2} T}{\partial z^{2}}\right)
\end{aligned}
$$

where $u$ and $w$ are radial and axial velocities along $r$ and $z$-axis, respectively, $\rho_{f}$ is the density of the nanofluid, $\sigma$ is the electrical conductivity, $v$ is the kinematic viscosity, $\tau=(\rho c)_{p} /(\rho c)_{f}$ is the ratio of the effective heat capacity of the nanoparticle material and the nanofluid, $\alpha$ is the thermal diffusivity, $D_{B}$ is the Brownian motion coefficient, $D_{T}$ is the thermophoretic diffusion coefficient, $T$ is the temperature of the fluid, $T_{m}$ is the mean fluid temperature and $C$ is the nanoparticles concentration.

The appropriate boundary conditions for the problem are 


$$
\begin{aligned}
& u=-\beta_{1} \frac{\partial u}{\partial z}, \quad w=\frac{w_{0}}{\sqrt{1-\alpha t}}, \quad T=-\gamma_{1} \frac{\partial T}{\partial z}+T_{w}, \quad C=C_{w} \quad \text { at } \quad z=0, \\
& u=\beta_{1} \frac{\partial u}{\partial z}, \quad w=w_{h}\left(=\frac{d h}{d t}\right), \quad T=\gamma_{1} \frac{\partial T}{\partial z}+T_{h}, \quad C=C_{h} \quad \text { at } \quad z=h(t)
\end{aligned}
$$

where $\beta_{1}$ and $\gamma_{l}$ are the velocity and thermal slip parameters, respectively. $T_{w}$ and $C_{w}$ denote the temperature and nanoparticles concentration at the lower disk while the temperature and nanoparticles concentration at the upper disk are $T_{h}$ and $C_{h}$, respectively.

Let us introduce the following dimensionless quantities [4]

$$
\begin{array}{ll}
\eta=\frac{z}{H \sqrt{1-\alpha t}}, & u=\frac{\alpha r}{2(1-\alpha t)} f^{\prime}(\eta), \quad w=-\frac{\alpha H}{2 \sqrt{1-\alpha t}} f(\eta), \\
\theta=\frac{T-T_{h}}{T_{w}-T_{h}}, & \phi=\frac{C-C_{h}}{C_{w}-C_{h}} .
\end{array}
$$

In view of Eqs (2.7), the continuity Eq.(2.1) is automatically satisfied while Eqs (2.2)-(2.5) after some mathematical simplification reduce to

$$
\begin{aligned}
& f^{\prime \prime \prime \prime}-S\left(\eta f^{\prime \prime \prime}+3 f^{\prime \prime}+f f^{\prime}-2 f f^{\prime \prime \prime}\right)-M^{2} f^{\prime \prime}=0 \\
& \theta^{\prime \prime}+\operatorname{Pr} S\left(2 f \theta^{\prime}-\eta \theta^{\prime}\right)+\operatorname{PrNb} \theta^{\prime} \phi^{\prime}+\operatorname{Pr} N t \theta^{2}=0 \\
& \phi^{\prime \prime}+\operatorname{Le} S\left(2 f \phi^{\prime}-\eta \phi^{\prime}\right)+\frac{N t}{\mathrm{Nb}} \theta^{\prime \prime}=0 .
\end{aligned}
$$

Also the boundary conditions (2.6) become

$$
\begin{aligned}
& f=A, \quad f^{\prime}+\beta f^{\prime \prime}=0, \quad \theta+\gamma \theta^{\prime}=1, \quad \phi=0 \quad \text { for } \quad \eta=0, \\
& f=\frac{1}{2}, \quad f^{\prime}-\beta f^{\prime \prime}=0, \quad \theta-\gamma \theta^{\prime}=0, \quad \phi=0 \quad \text { for } \quad \eta=1 .
\end{aligned}
$$

Here $S=\frac{\alpha H^{2}}{2 v}$ is the squeeze number where $S>0$ corresponds to the plates moving apart while $S<0$ corresponds to the plates moving together (the so called squeezing flow), $M=H B_{0} \sqrt{\frac{\sigma}{\mu}}$ is the Hartmann parameter, $\operatorname{Pr}=\frac{v}{\alpha}$ is the Prandtl number, $\mathrm{Le}=\frac{v}{D_{B}}$ is the Lewis number, $N b=\frac{\tau D_{B}\left(C_{w}-C_{h}\right)}{v}$ is the Brownian motion parameter, $\mathrm{Nt}=\frac{\tau D_{T}\left(T_{w}-T_{h}\right)}{v T_{m}}$ is the thermophoresis parameter, $A=\frac{w_{0}}{\alpha H}$ is the suction/injection parameter, $\beta=\frac{\beta_{1}}{H \sqrt{1-\alpha t}}$ is the dimensionless velocity slip parameter and $\gamma=\frac{\gamma_{1}}{H \sqrt{1-\alpha t}}$ is the dimensionless temperature slip parameter. 
The most important characteristics of flow, heat and mass transfer are the reduced skin friction coefficient $\mathrm{C}_{\text {fr }}$, the reduced Nusselt number $\mathrm{Nu}$ and the reduced Sherwood Sh that are defined as

$$
\begin{aligned}
& \mathrm{C}_{f r}=f^{\prime \prime}(1) \quad \text { where } \quad \mathrm{C}_{f r}=\frac{H^{2} \sqrt{1-\alpha t} \mathrm{Re}_{r}}{r^{2}} C_{f}, \\
& \mathrm{Nur}=-\theta^{\prime}(1) \quad \text { where } \quad \mathrm{Nur}=\sqrt{1-\alpha t} \mathrm{Nu}, \\
& \text { and } \quad \mathrm{Shr}=-\phi^{\prime}(1) \quad \text { where } \quad \mathrm{Shr}=\sqrt{1-\alpha t} \mathrm{Sh}
\end{aligned}
$$

where $\operatorname{Re}_{r}=\frac{r \alpha H}{2 v}$ is the local squeeze Reynolds number.

It is worth mentioning that in the absence of slip velocity (i.e., $\beta=0$ ) and slip temperature (i.e., $\gamma=0$ ), the boundary conditions (2.11) reduce to (2.10) of Hashmi et al. [18].

\section{Method of solution}

The set of Eqs (2.8), (2.9) and (2.10) under the boundary conditions (2.11) have been solved numerically by applying the shooting iteration technique together with the sixth-order Runge-Kutta integration scheme. The unspecified initial conditions are assumed and then integrated numerically as an initial valued problem to a given terminal point. Improvement is made on the values of assumed missing initial conditions by iteratively comparing the calculated value of the dependent variable at the terminal point with its given value there. The computations are done by a written program which uses a symbolic and computational computer language MAPLE.

In order to ascertain the accuracy of our numerical results, the present study (in the absence of slip parameter) is compared with those of Hashmi et al. [18]. The values of Nur and Shr have been calculated for various values of $\mathrm{Nb}$ and $\mathrm{Nt}$. An excellent agreement is found between the two set of results as shown in Tab.1. Thus the use of the present numerical code for the current model is justified.

Table 1. Comparison of the values of the local Nusselt number and local Sherwood number for various values of $\mathrm{Nb}$ and $\mathrm{Nt}$ with $\beta=\gamma=0$.

\begin{tabular}{cccccc}
\hline $\mathrm{Nb}$ & $N t$ & \multicolumn{2}{c}{ Nur } & \multicolumn{2}{c}{ Shr } \\
\hline & & Hashmi et al. $[18]$ & Present method & Hashmi et al. [18]. & \multicolumn{1}{c}{ Present method } \\
\cline { 3 - 6 } 0.1 & 0.1 & 0.52628540 & 0.526285397692707 & 0.86604666 & 0.866046665141329 \\
0.5 & & 0.63433253 & 0.634332530012476 & 0.53012814 & 0.530128143896286 \\
1.0 & & 0.78636385 & 0.786363850155776 & 0.48603919 & 0.486039186120291 \\
1.5 & & 0.95569955 & 0.955699547716439 & 0.46986157 & 0.469861566526085 \\
1.5 & 0.5 & 1.17682119 & 1.176821184883260 & 0.40180718 & 0.401807177532398 \\
& 1.0 & 1.48581207 & 1.485811936635642 & 0.12619334 & 0.126193335885242 \\
& 1.5 & 1.82305276 & 1.823053529110100 & 0.39083080 & 0.390839865635371 \\
& 2.0 & 2.17915991 & 2.179227931099257 & 1.16777723 & 1.16800852390237 \\
\hline
\end{tabular}

\section{Results and discussions}

In order to get an insight into the effects of different parameters of practical importance on the flow characteristics, the numerical results are presented graphically in Figs 2-16 for several sets of values of the 
pertinent parameters. In the simulation the default values of the parameters are considered as $A=2, S=1, N t=$ $\mathrm{Nb}=0.2, \beta=0.05, \gamma=0.1, M=0.5, \mathrm{Le}=\operatorname{Pr}=1.0$ unless otherwise specified.

The effect of the magnetic field parameter $M$ on the radial velocity is depicted in Fig.2. It is observed from the figure that the fluid velocity decreases with an increase in the values of $M$ and so increases the thickness of momentum boundary layer near the wall region, i.e., for $\eta<0.2$ and $\eta>0.7$ (not precisely determined). The reason behind this phenomenon is that the application of a magnetic field to an electrically conducting nanofluid gives rise to a resistive type force called the Lorentz force. This force has the tendency to slow down the motion of the nanofluid in the boundary layer region. However, the opposite trend is observed in the central region, i.e., for $0.2 \leq \eta \leq 0.7$ (not precisely determined) due to mass conservation. Figure 3 shows the influence of the slip parameter $\beta$ on the radial velocity. The figure clearly indicates that in the absence of the slip velocity, i.e., when $\beta=0$, the radial velocity of the fluid at the disks' surfaces is the same as that of the disks' surfaces, which is zero in the present case. Also, it is observed that the magnitude of the radial velocity acquires a positive value on the disks' surface in the presence of the slip velocity which increases with the increase in the slip parameter $\beta$ for $\eta<0.2$ and $\eta>0.8$ (not precisely determined). But the effect is totally reversed i.e., the fluid velocity decreases with an increase in the values of the slip parameter $\beta$ for $0.2 \leq \eta \leq 0.8$ (not precisely determined). The physics behind this is that the increased slip parameter near the wall region decreases the velocity gradient. Also, an increase in the fluid velocity near the wall will be compensated by a corresponding decrease in the fluid velocity near the central region as the mass flow rate is kept constant. With the increase in the squeeze number $S$, the fluid velocity decreases at first near the lower disk region, i.e., for $\eta<0.4$ (not precisely determined); then it starts increasing with the increase in $S$ near the upper disk region as shown in Fig.4. These results are in a good agreement with the results obtained by Hashmi et al. [18] in the absence of slip parameters.

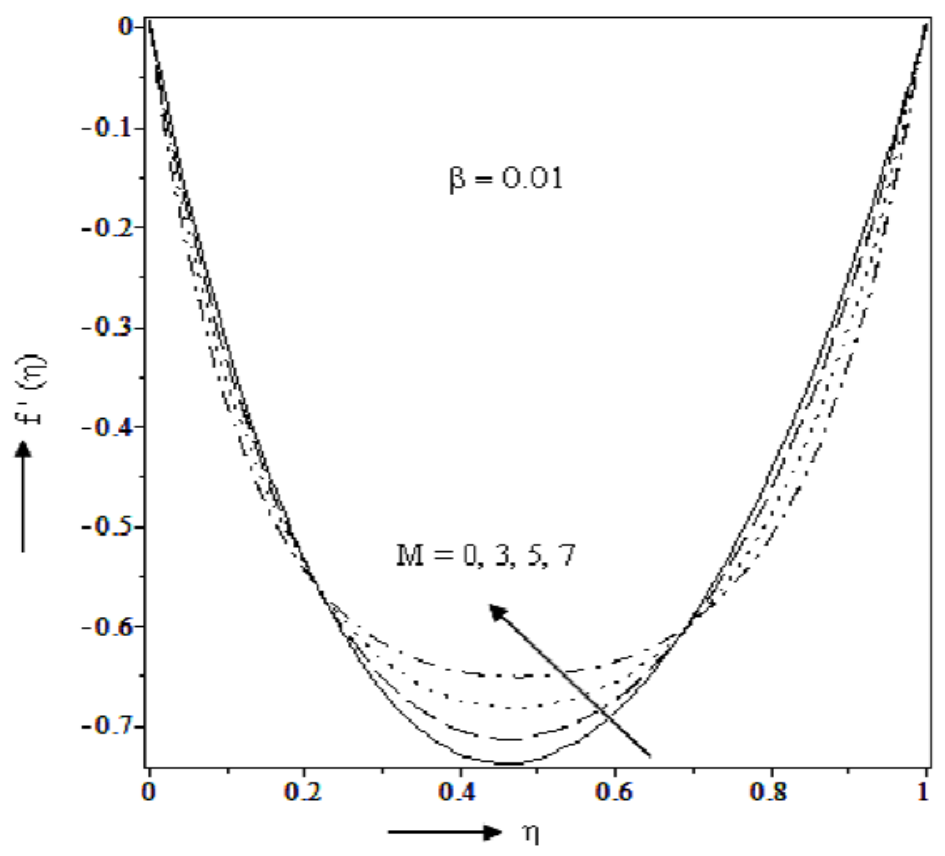

Fig.2. Velocity profiles for various values of $M$. 


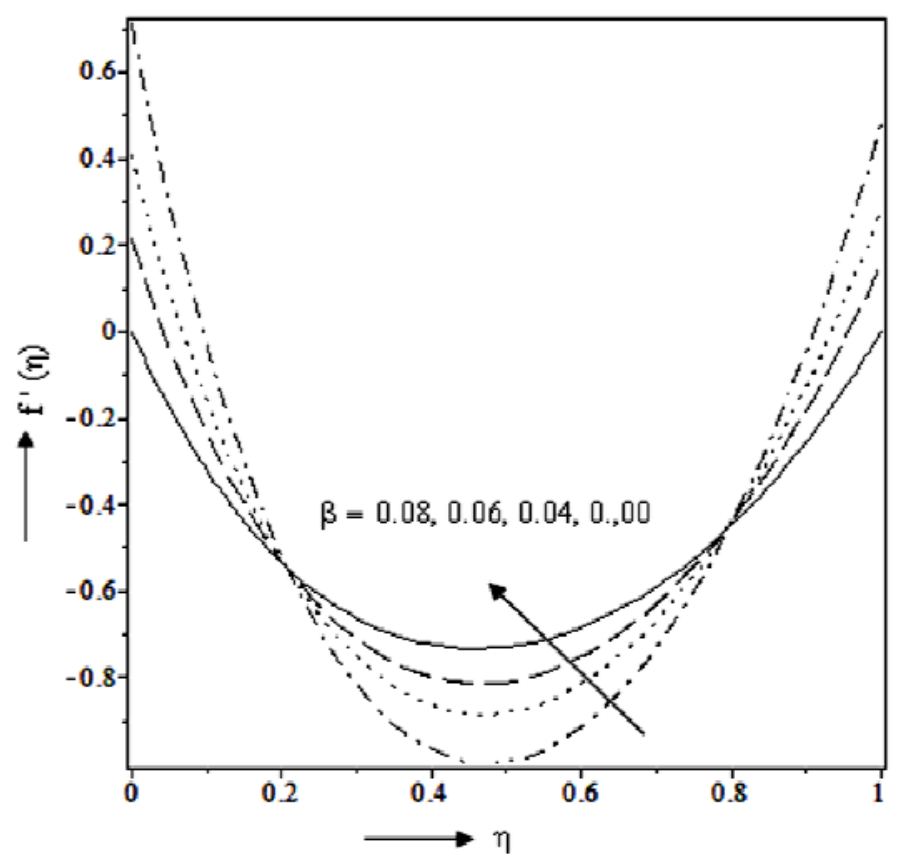

Fig.3. Velocity profiles for various values of $\beta$.

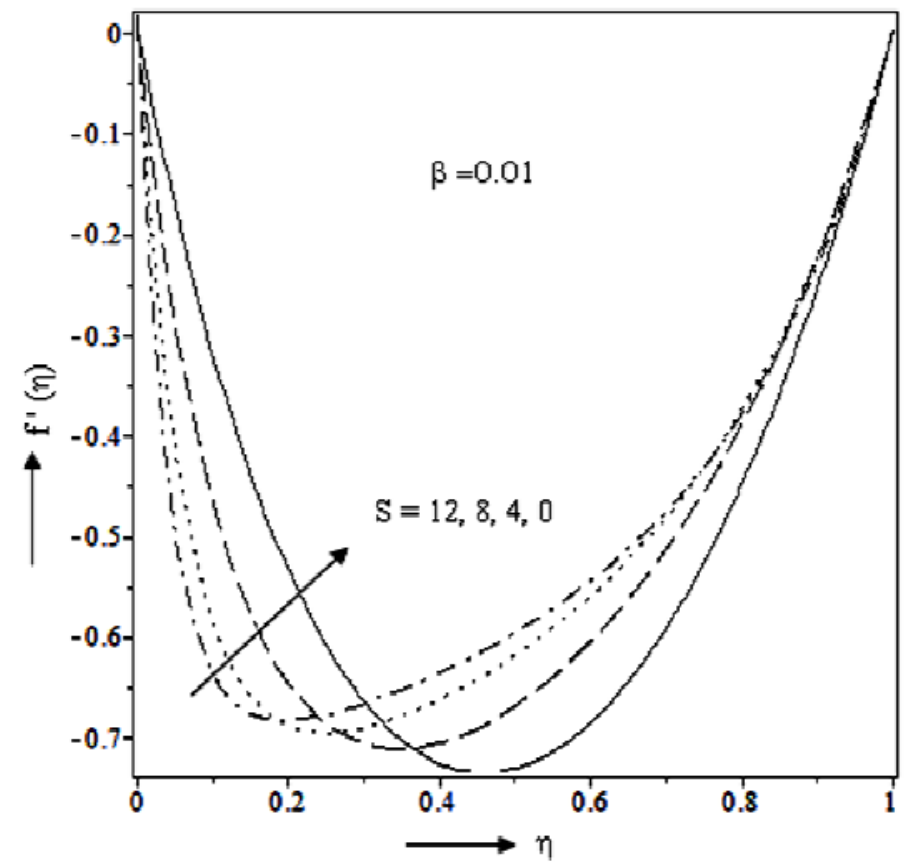

Fig.4. Velocity profiles for various values of $S$.

Figure 5 demonstrates the effects of the thermal slip parameter $\gamma$ on the fluid temperature in the presence of suction. It is observed from the figure that in the absence of the thermal slip parameter, i.e., when $\gamma=0$, the temperature of the fluid and that of the disks' surfaces is same, which in the present problem is 1 and 0 for lower and upper disks, respectively. As the slip parameter $\gamma$ increases, the temperature of the fluid increases near the lower disk surface while a reverse behaviour occurs near the upper disk surface. The effects of the parameters controlling the Brownian motion and thermophoresis on the temperature distribution are well presented in Figs 6, 7 in the presence of thermal slip. It is observed from the figures that the temperature of the fluid decreases at first with an increase in both the values of the Brownian motion parameter $\mathrm{Nb}$ and thermophoresis parameter $N t$ for $\eta<0.2$ (not precisely determined); then it starts increasing 
near the central region, i.e., for $0.2 \leq \eta \leq 0.8$ (not precisely determined) and then it again decreases with increasing both the values of $\mathrm{Nb}$ and $N t$ near the upper disk region i.e., for $\eta>0.8$ (not precisely determined). An effect of varying the value of the squeeze number $S$ on the temperature distribution is illustrated in Fig.8. With the increase in the squeeze number $S$, the temperature of the fluid decreases near the lower disk region i.e., for $\eta<0.8$ (not precisely determined) but a reverse effect occurs near the upper disk region. It is worth mentioning that an increase in $S$ can be associated with a decrease in the kinematic viscosity, an increase in the distance between the plates and an increase in the speed at which the plates move. These results are found to be identical to the results of Hashmi et al. [18] in the absence of slip parameters.

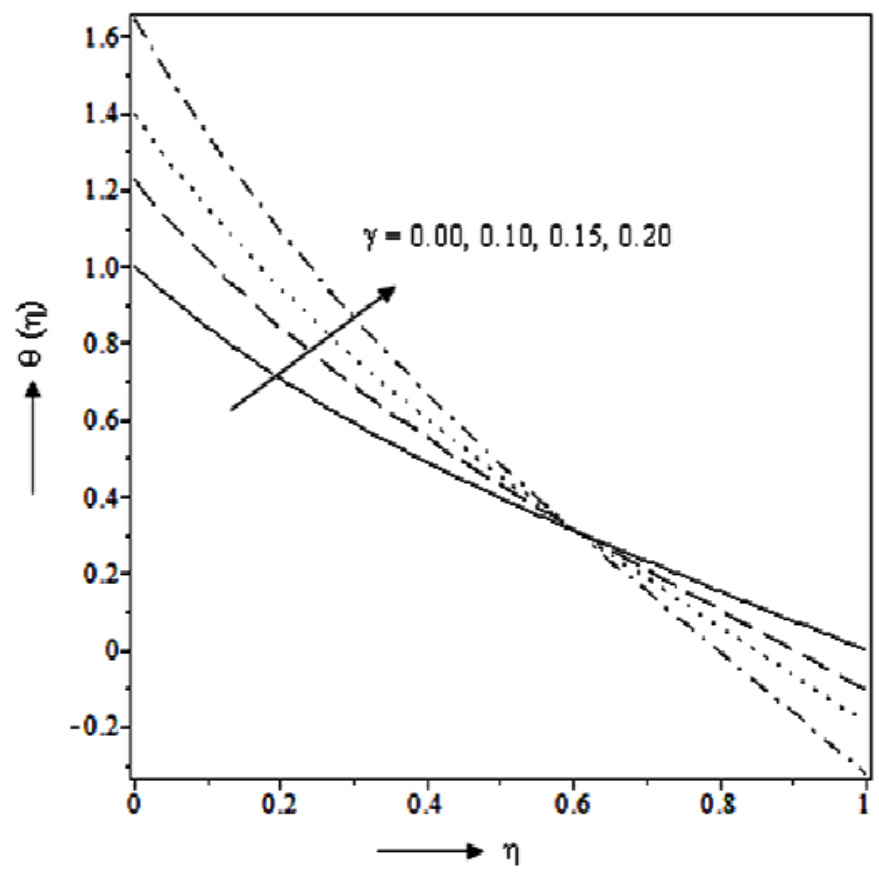

Fig.5. Temperature profiles for various values of $\gamma$.

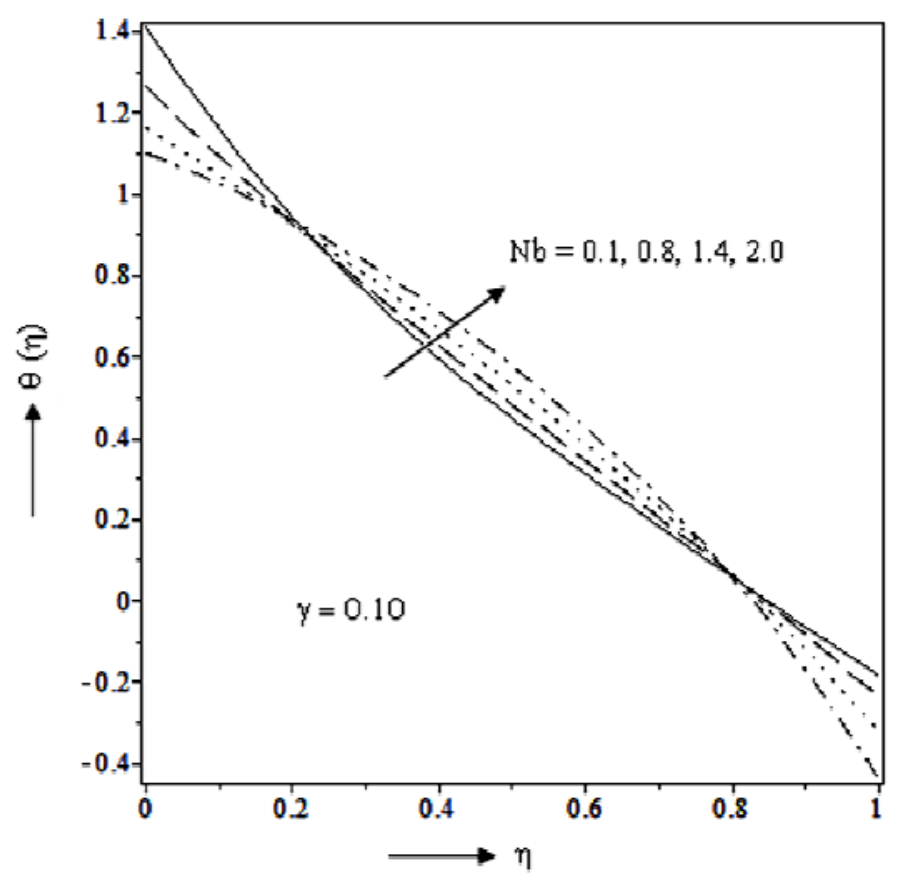

Fig.6. Temperature profiles for various values of $\mathrm{Nb}$. 


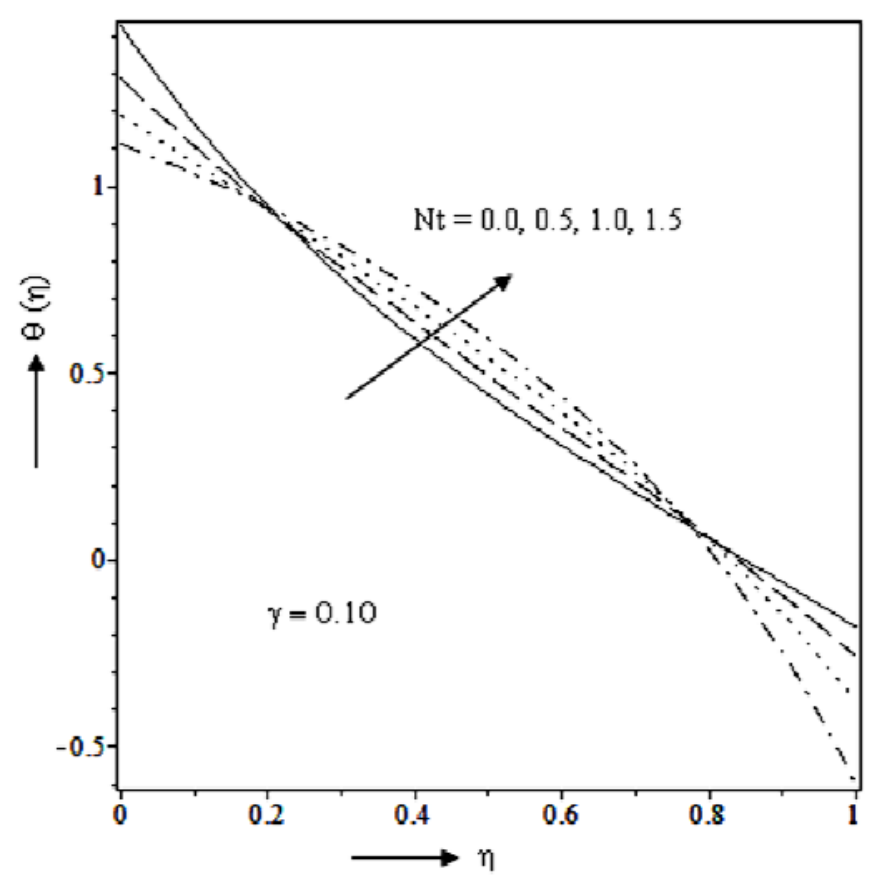

Fig.7. Temperature profiles for various values of $N t$.

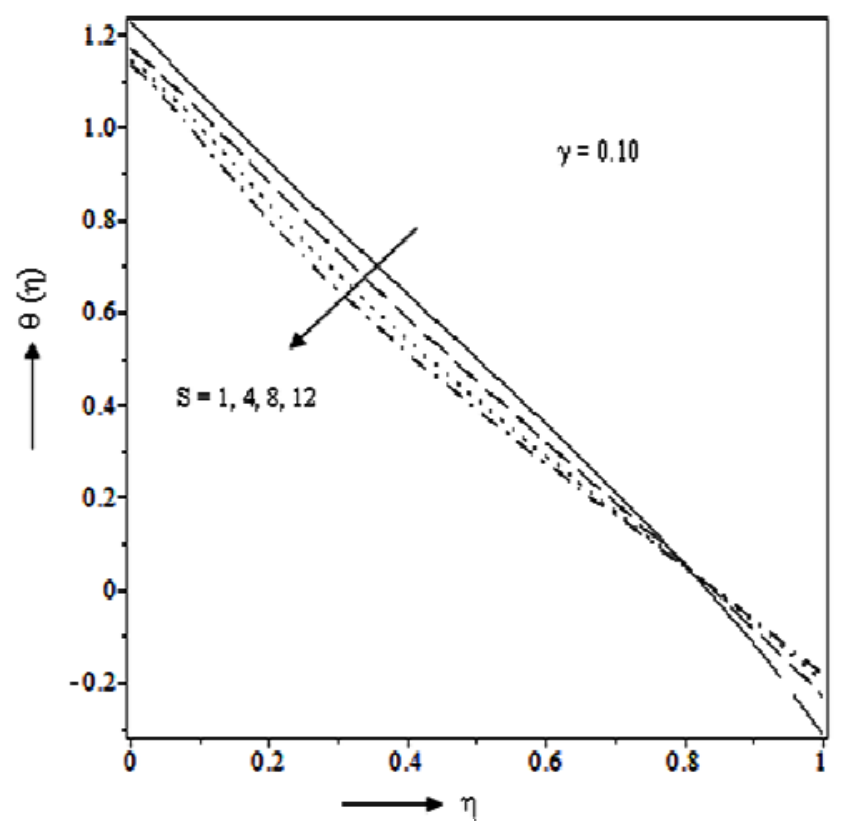

Fig.8. Temperature profiles for various values of $S$.

Figure 9 shows the variation of the nanoparticles concentration distribution for different values of the Lewis number Le in the presence of suction. It is seen that the concentration decreases with the increase in the values of Le. Also, it is observed that the concentration of the fluid decreases drastically for higher values of Le near the lower disk region. The impact of the thermophoresis on the concentration distribution is presented in Fig.10. It is seen that the effect of increasing the values of the thermophoresis parameter $N t$ results in increasing concentration distribution rapidly near the wall region of the lower disk whereas the 
concentration decreases steadily from the lower disk region to the upper disk region in the absence of thermophoresis. Figure 11 reveals that the nanoparticles concentration decreases uniformly with increasing the values of the Brownian motion parameter $\mathrm{Nb}$. These results fall in line with the results obtained by Hashmi et al. [18] in the absence of the slip parameter. The concentration distribution decreases significantly with increasing the values of squeeze number $S$ as depicted in Fig. 12 in the presence of suction.

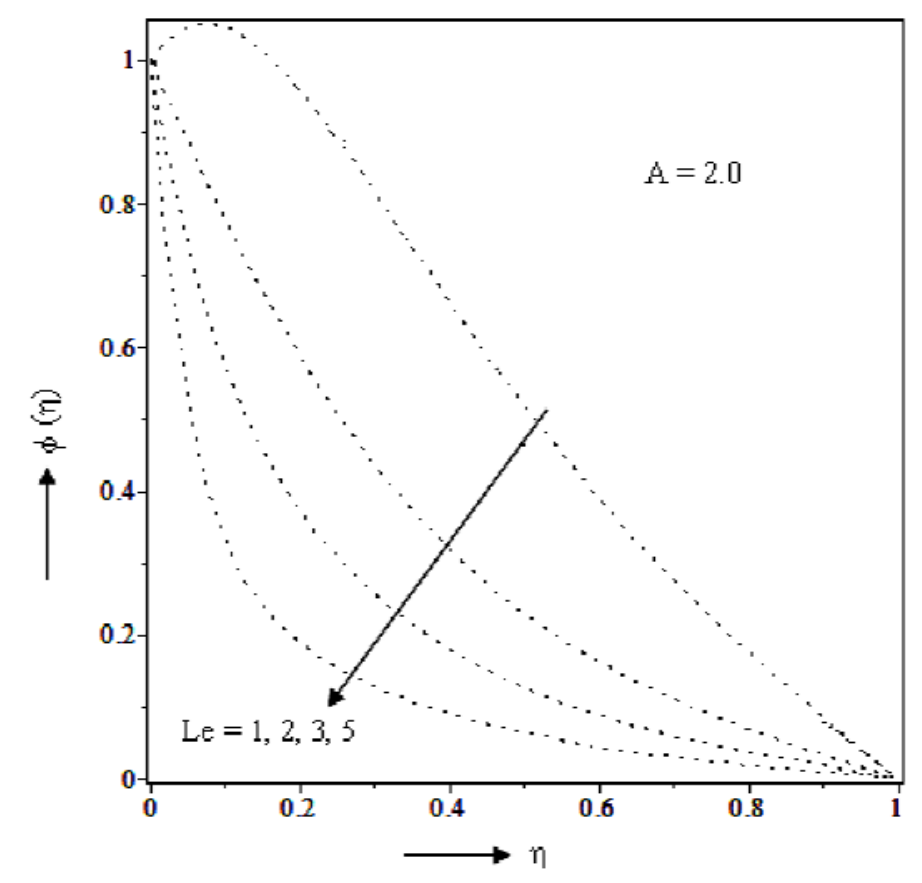

Fig.9. Concentration profiles for various values of Le.

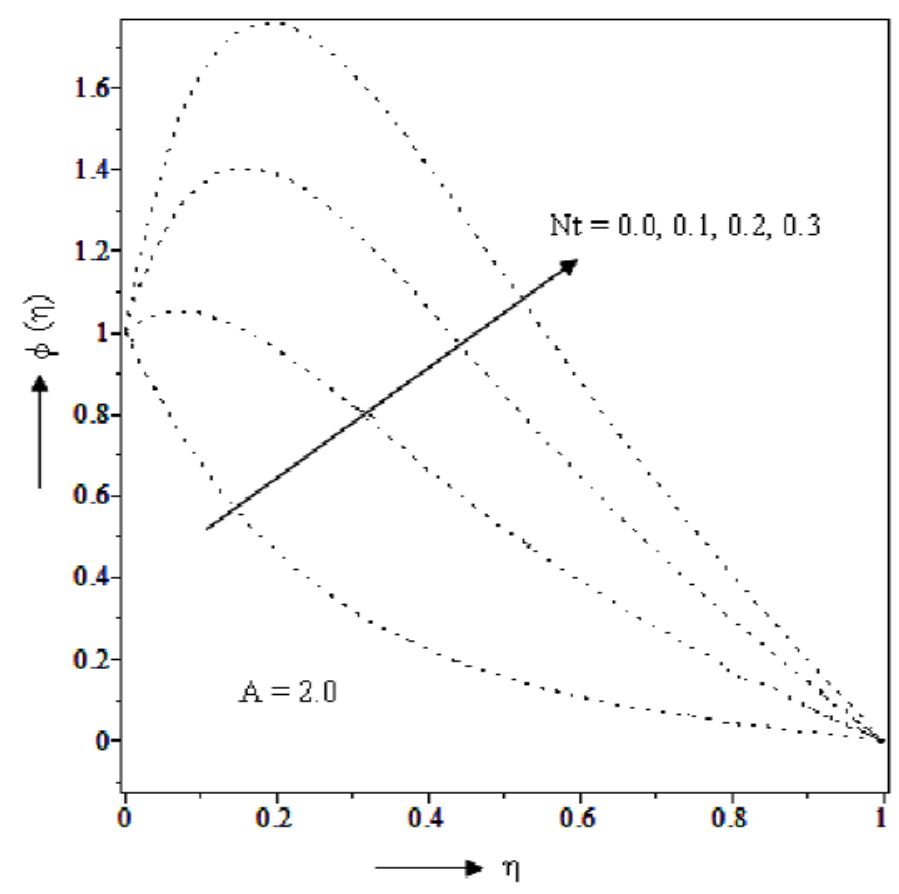

Fig.10. Concentration profiles for various values of $N t$. 


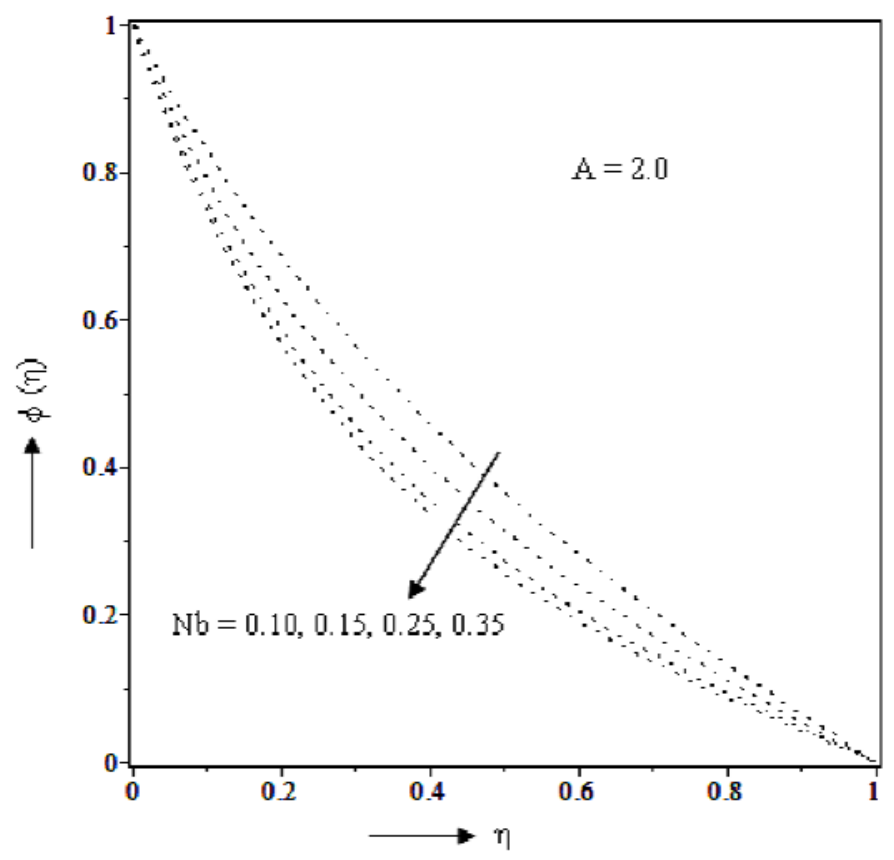

Fig.11. Concentration profiles for various values of $\mathrm{Nb}$.

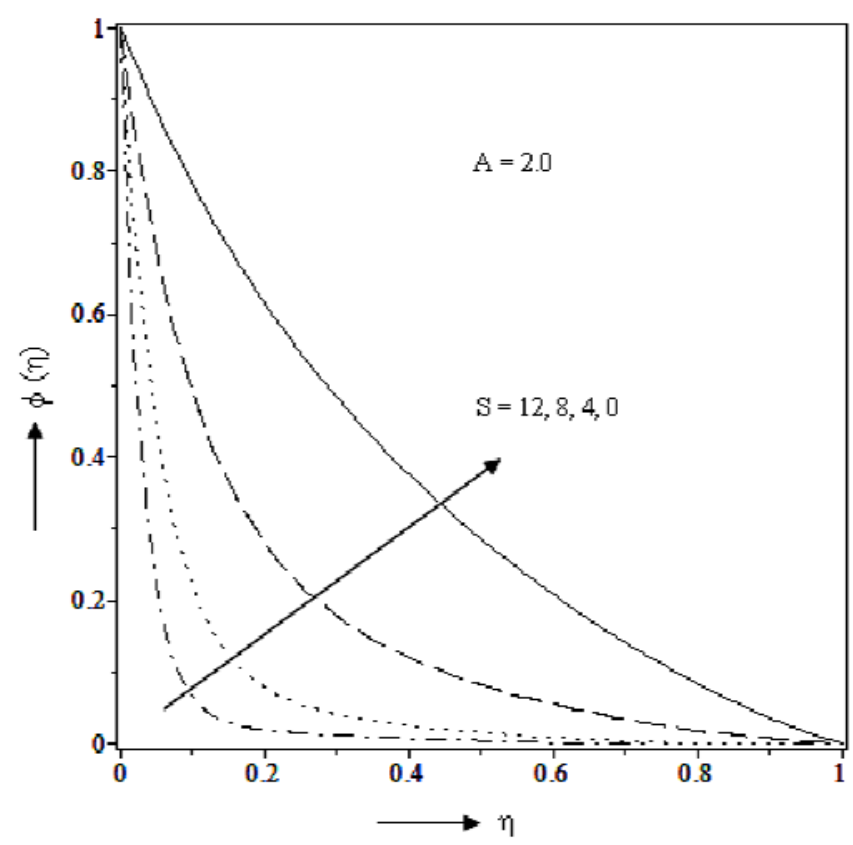

Fig.12. Concentration profiles for various values of $S$.

Figures 13, 14 show variation of the skin friction coefficient on the wall of the upper disk versus the squeeze number $S$ for different values of the magnetic field parameter $M$ and slip parameter $\beta$, respectively, in the presence of suction. A combination of different applications which appear in these figures guides us to understand carefully how the skin friction coefficient on the wall of the upper disk change. Increasing $S$ 
tends to decrease the skin friction coefficient for different values of $M$ and $\beta$ but the effect is not significant for higher values of $\beta$. On the other hand, the magnetic field parameter $M$ increases the skin friction coefficient on the upper disk wall whereas the slip parameter $\beta$ moves it down to reach a lower value. Figure 15 illustrates the variation of the Nusselt number at the upper disk wall versus the thermophoresis parameter $N t$ for various values of the surface thermal slip parameter $\gamma$. This figure indicates that the Nusselt number increases with the increasing thermophoresis parameter $N t$. But for a large temperature slip parameter $\gamma$, the increase in $N t$ has a prominent effect on the dimensionless heat transfer rate. It is also observed that the increase of $\gamma$ results in an increase in the Nusselt number. Thus the temperature slip parameter has a significant effect on heat transfer due to an increased temperature gradient on the upper disk wall. Figure 16 displays that the rate of mass transfer, i.e., the Sherwood number decreases considerably as the Lewis number Le increases whereas an opposite effect is observed for the thermophoresis parameter $N t$. Thus the rate of mass transfer increases actively due to the presence of thermophoresis.

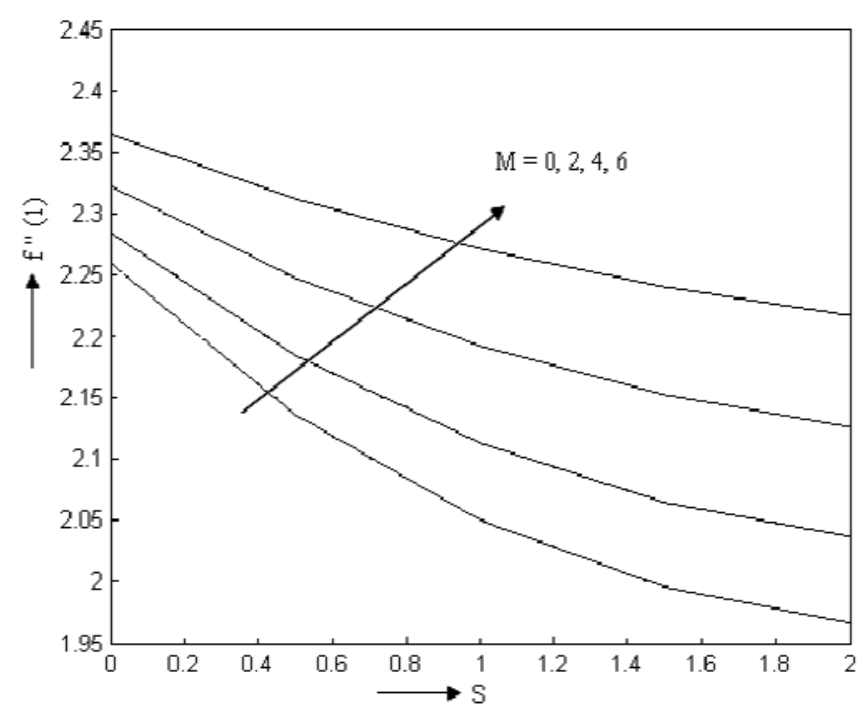

Fig.13. Effects of $M$ and $S$ on the skin friction coefficient.

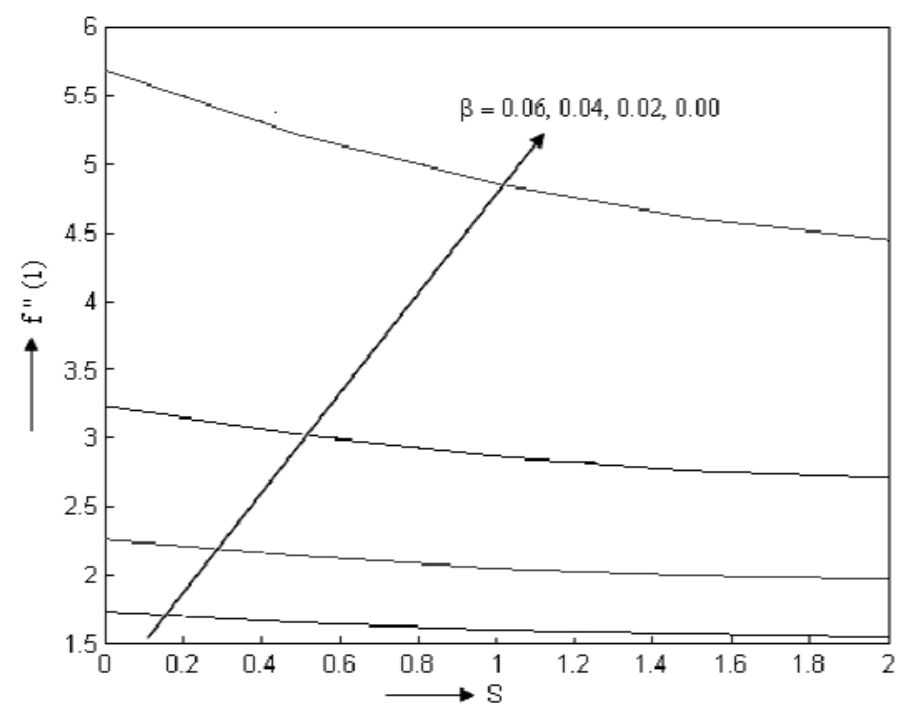

Fig.14. Effects of $\beta$ and $S$ on the skin friction coefficient. 


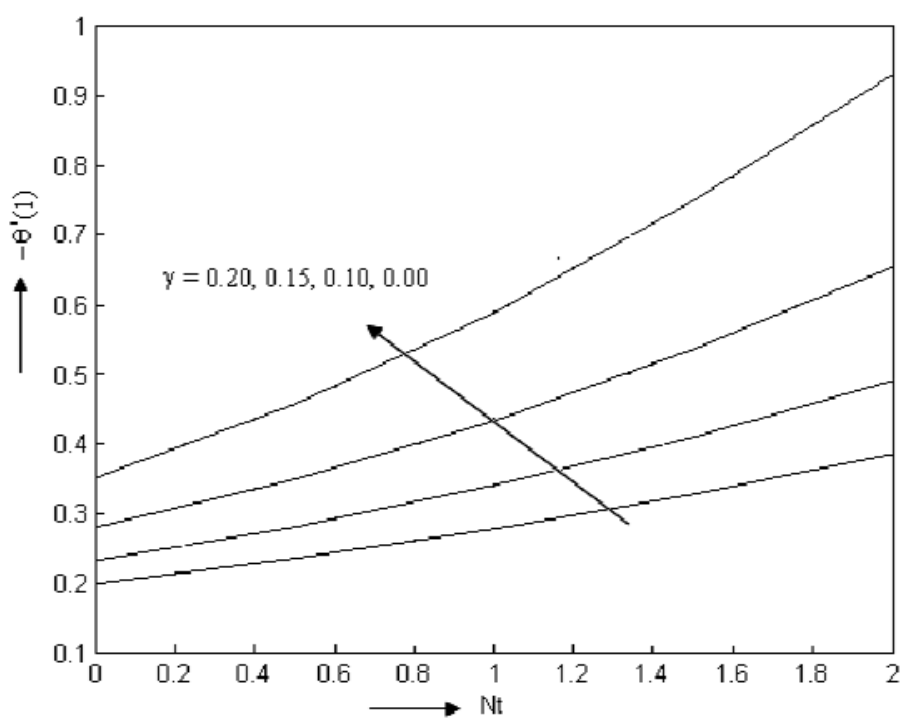

Fig.15. Effects of $\gamma$ and $N t$ on the Nusselt number.

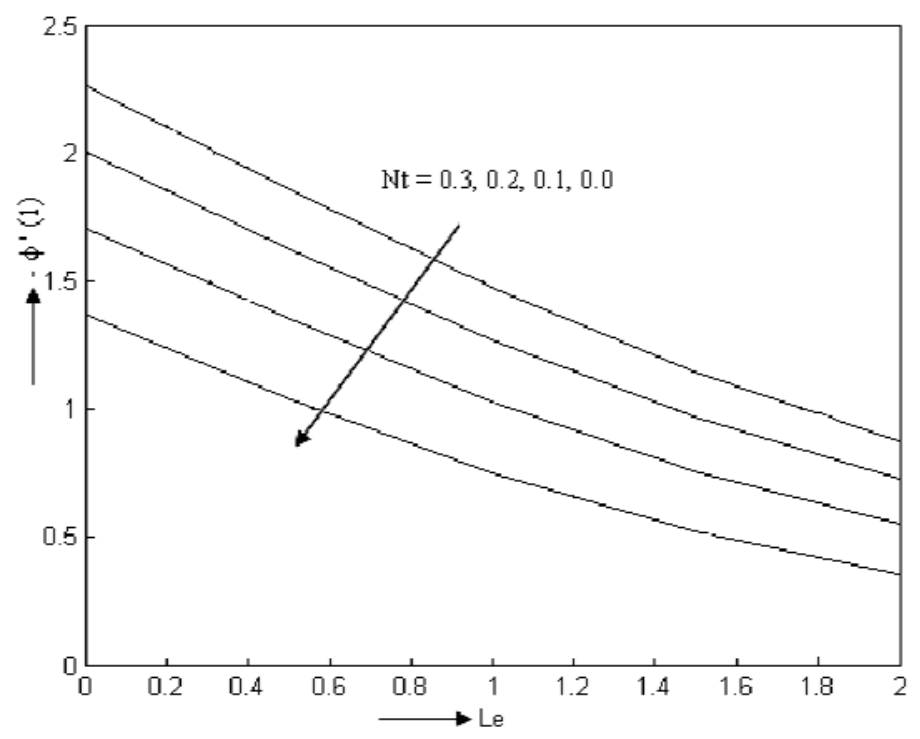

Fig.16. Effects of $N t$ and Le on the Sherwood number.

\section{Conclusions}

In the present study, the influence of velocity and thermal slip conditions on the unsteady MHD squeezing flow in a viscous, incompressible and electrically conducting nanofluid between two parallel disks is analyzed using a numerical technique. The Nachtsheim and Swigert shooting iteration technique together with the Runge -Kutta sixth-order integration scheme is used to obtain the numerical solution of the nonlinear flow problem. Numerical results are presented through graphs to illustrate the details of the flow characteristics and their dependence on material parameters. The results presented here are potentially in controlling wall shear stress as well as the Nusselt and the Sherwood number. Also, the present study has a direct association with nanofluidics and microscale flows where the no-slip boundary 
condition usually breaks down. Some important positive effects of the slip in nanofluidics are the friction reduction, energy conservation and micromixing of biological samples under a magnetic field in microchannels.

\section{Nomenclature}

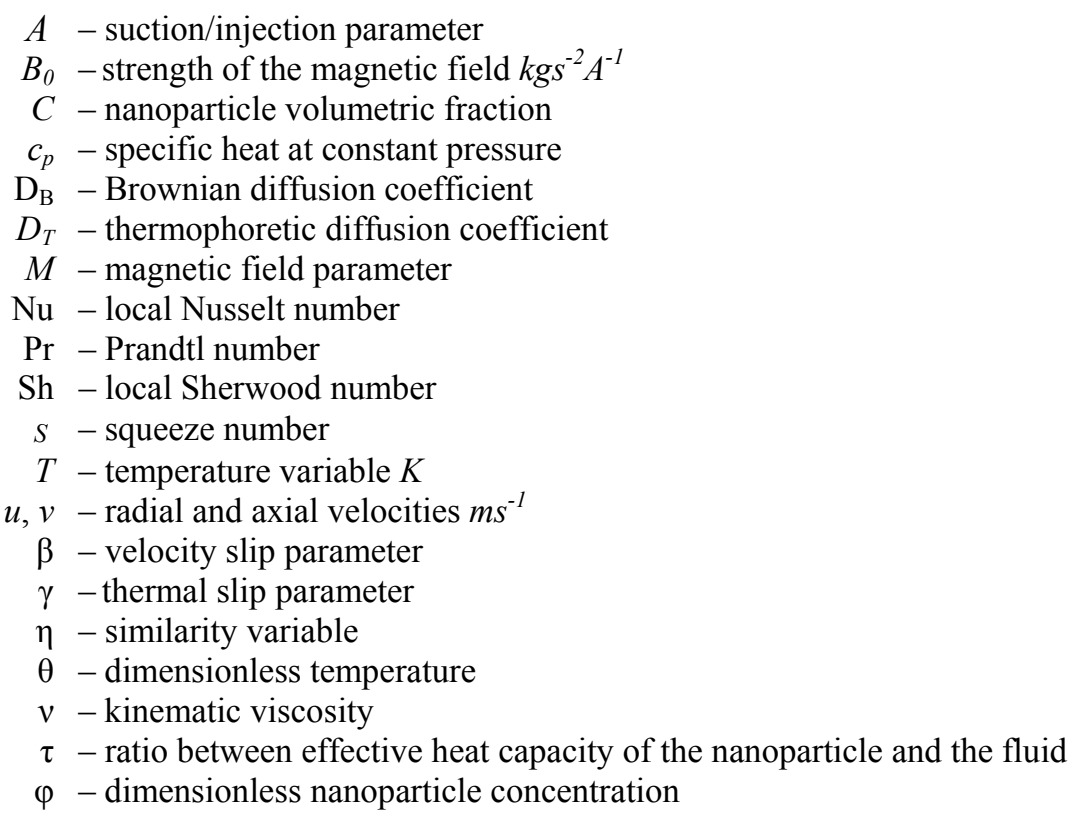

\section{Subscript}

Le - Lewis number

$\mathrm{Nb}$ - Brownian motion parameter

$N t$ - thermophoresis parameter

$w$ - condition on the sheet

- - differentiation with respect to $\eta$

\section{References}

[1] Stefan M.J. (1874): Versuch über die scheinbare Adhäsion, Sitzungsber. - Abt. II, Österr. Akad. Wiss., Math.Naturwiss. K1., vol.69, pp.713-721.

[2] Rashidi M.M., Shahmohamadi H. and Dinarvand S. (2008): Analytic approximate solutions for unsteady twodimensional and axisymmetric squeezing flows between parallel plates. - Mathematical Problems in Engineering, vol.2008, pp.935095.

[3] Siddiqui A.M., Irum S. and Ansari A.R. (2008): Unsteady squeezing flow of a viscous MHD fluid between parallel plates. - Mathematical Modelling and Analysis, vol.13, pp.565-576.

[4] Domairry G. and Aziz A. (2009): Approximate analysis of MHD Squeeze flow between two parallel disks with suction or injection by homotopy perturbation method. - Mathematical Problems in Engineering, vol.2009, pp.603916.

[5] Haya T., Yousaf A., Mustafa M. and Obaidat S. (2011): MHD squeezing flow of second-grade fluid between two parallel disks. - International Journal of Numerical Method in Fluids, vol.69, pp.399-410.

[6] Mustafa M., Hayat T. and Obaidat S. (2012): On heat and mass transfer in the unsteady squeezing flow between parallel plates. - Meccanica, vol.47, pp.1581-1589. 
[7] Choi S.U.S. (1995): Enhancing thermal conductivity of fluids with nanoparticles. - Devlopment and Applications of Non-Newtonian Flows, vol.66, pp.99-105.

[8] Choi S.U.S., Zhang Z.G., Yu W., Lockwood F.E. and Grulke E.A. (2001): Anomalously thermal conductivity enhancement in nanotube suspensions. - Applied Physics Letters, vol.79, pp.2252-2254.

[9] Buongiorno J. (2006): Convective transport in nanofluids. - ASME Journal of Heat Transfer, vol.128, pp. $240-250$.

[10] Kuznetsov A.J. and Nield N.D. (2010): Natural convective boundary layer flow of a nanofluid past a vertical plat. - International Journal of Thermal Sciences, vol.49, pp.243-247.

[11] Khan W.A. and Aziz A. (2011): Natural convection flow of a nanofluid over a vertical plate with uniform surface heat flux. - International Journal of Thermal Sciences, vol.50, pp.1207-1207.

[12] Khan W.A. and Aziz A. (2011): Double-diffuusive natural convective boundary layer flow in a porous medium saturated with a nanofluid over a vertical plate, prescribed surface heat, solute and nanoparticle fluxes. International Journal of Thermal Sciences, vol.50, pp.2154-2160.

[13] Yao S., Fang T. and Zhong Y. (2011): Heat transfer of a generalized stretching/shrinking wall problem with convective boundary conditions. - Communications in Nonlinear Science and Numerical Simulation, vol.16, pp.752-760.

[14] Makinde D. and Aziz A. (2011): Boundary layer flow of a nanofluid past a stretching sheet with a convective boundary condition. - International Journal of Thermal Sciences, vol.50, pp.1326-1332.

[15] Aziz A. and Khan W.A. (2012): Natural convective boundary layer flow of a nanofluid past a convectively heated vertical plate. - International Journal of Thermal Sciences, vol.52, pp.83-90.

[16] Hamad M.A.A. and Ferdows M. (2012): Similarity solution of boundary layer stagnation-point flow towards a heated porous stretching sheet saturated with a nanofluid with heat absorption/generation and suction/blowing: A lie group analysis. - Communication in Nonlinear Science and Numerical Simulatation, vol.17, pp.132-140.

[17] Kandasamy R., Loganathanb P. and Puvi Arasub P. (2011): Scaling group transformation for MHD boundarylayer flow of a nanofluid past a vertical stretching surface in the presence of suction/injection. - Nuclear Engineering and Design, vol.241, pp.2053-2059.

[18] Hashmi M.R., Hayat T. and Alsaedi A. (): On the analytic solutions for squeezing flow of nanofluids between parallel disks. - Nonlinear analysis: Modelling and Control, vol.17, No.4, pp.418-430.

[19] Das K. (2013): Lie group analysis of stagnation point flow a nanofluid. - Microfluidics and Nanofluidics, vol.15, pp.267-274.

[20] Navier C.L.M.H. (1823): Mémoire sur les lois du mouvement des fluids. - Mém Acad Roy Sci Inst France, vol.6, pp.389-440.

[21] Shikhmurzaev Y.D. (1993): The moving contact line on a smooth solid surface. - International Journal of Multiphase Flow, vol.19, pp.589-610.

[22] Choi C.H., Westin J.A. and Breuer K.S. (2002): To slip or not to slip water flows in hydrophilic and hydrophobic microchannels. - In: Proceedings of IMECE 2002, New Orlaneas, LA, Paper No. 2002-33707.

[23] Martin M.J. and Boyd I.D. (2006): Momentum and heat transfer in a laminar boundary layer with slip flow. Journal of Thermophysics and Heat Transfer, vol.20, No.4, pp.710-719.

[24] Matthews M.T. and Hill J.M. (2007): Nano boundary layer equation with nonlinear Navier boundary condition. Journal of Mathematical Analysis and Application, vol.333, pp.381-400.

[25] Ariel P.D. (2007): Axisymmetric flow due to a stretching sheet with partial slip. - Computer and Mathematics with Applications, vol.54, pp.1169-1183.

[26] Wang C.Y. (2009): Analysis of viscous flow due to a stretching sheet with surface slip and suction. - Nonlinear Analysis: Real World Application, vol.10, No.1, pp.375-380.

[27] Das K. (2012): Impact of thermal radiation on MHD slip flow over a flate plate with variable fluid properties. Heat Mass and Transfer, vol.48, pp.767-778. 
[28] Das K. (2012): Slip effects on heat and mass transfer in MHD micropolar fluid flow over an inclined plate with thermal radiation and chemical reaction. - International Journal of Numerical Method in Fluids, vol.70, pp.96-11.

[29] Hussain A., Mohyud- Cheema S.T. and Din T.A. (2012): Analytical and Numerical Approaches to Squeezing Flow and Heat Transfer between Two Parallel Disks with Velocity Slip and Temperature Jump. - China Physics Letter, vol.29, pp.114705-1-5.

[30] Das K. (2012): Slip flow and convective heat transfer of nanofluids over a permeable stretching surface. Computers and Fluids, vol.64, No.1, pp.34-42.

Received: February 23, 2015

Revised: December 25, 2015 